\title{
Microstructural Characterization of Cast Alnico 8 Alloys
}

\author{
L. Zhou ${ }^{1}$, H. M. Dillon ${ }^{1}$, R. W. McCallum ${ }^{1}$, I. E. Anderson ${ }^{1}$, M. Kramer ${ }^{1}$, S. Constantinides ${ }^{2}$ \\ ${ }_{1}^{1}$ Ames Lab (USDOE), Iowa State University, Ames, IA 50011 \\ 2 Arnold Magnetic Technologies Corp., 770 Linden Avenue, Rochester, NY 14625
}

Concern for the supply of rare earth metals has stimulated the search for alternative magnetic materials. Alnico, with excellent magnetic stability at high temperature, is a promising candidate which was optimized prior to advent of todays advanced characterization tools [1]. Key to the optimization of the magnetic properties is control of the spinodal decomposition (SD) into a nonmagnetic phase and an FeCo-rich hard magnetic phase. Thus, understanding the structural relationship between those two phases, as well as influence on processing and chemistry are critical for improving properties of alnico alloys.

This study focuses on structural characterization of cast alnico 8 alloy from Arnold Magnetic Technologies. A combination of TEM techniques, including diffraction contrast TEM, high resolution transmission electron microscopy (HREM), high-angle annular-dark-field (HAADF) scanning transmission electron microscopy (STEM), energy dispersive X-ray spectroscopy, Lorentz microscopy and off-axis holography were used. Figure 1 (a) is a HAADF STEM image of alnico 8 along transverse direction showing a nanometer scale mosaic structure formed by SD. The bright region corresponds to $\mathrm{Fe}-\mathrm{Co}$ based bcc phase, while the dark region corresponds to AlNi-based L2 $2_{1}$ phase. The bcc phase facets on $\{110\}$ and $\{100\}$ planes. The small bright particles are $\mathrm{Cu}$ sitting at the corner of the bcc phase were also observed. Selected area diffraction pattern taken along [110] zone axis confirms that the AlNi-phase is the ordered L2 structure. Fig 1 (b) is a HAADF STEM image of alnico 8 looking along longitudinal direction. Orthogonally oriented elongated bec rods were observed.

Figure 2 (a, d) shows HAADF STEM images of two alnico 8 grains with different SD morphology. The grains are oriented differently relative to magnetic field direction applied during annealing process, as shown by the orientation imaging microscopy (OIM) pole figures (Fig. 2(c, f)). Lorentz microscopy images (Fig. 2(b, e)) show that the difference in SD morphology can cause a micro-magnetic domain morphology difference. Combination of OIM and TEM imaging indicated that bcc rods tends to dominantly elongate along the <001> direction with largest projected magnetic field.

Research was supported by U .S. DOE, Office of Energy Efficiency and Renewable Energy (EERE), under its Vehicle Technologies Program, through the Ames Laboratory, Iowa State University under contract DE-AC02-07CH11358.

[1] M. J. Kramer, R. W. McCallum, I. E. Anderson, and S. Constantinides, JOM , 64, 752, (2012). 

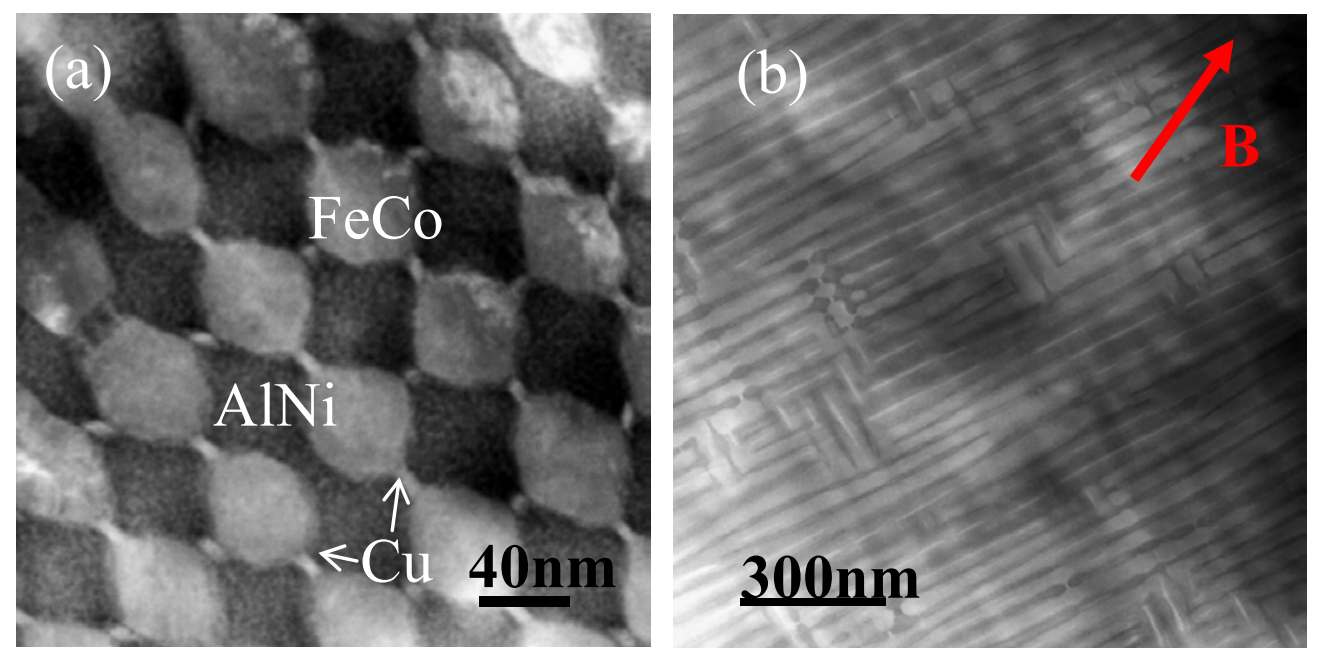

Figure 1. HAADF STEM images of alnico 8 along transverse (a) and longitudinal (b) direction. The applied magnetic field direction was indicated by red arrow.
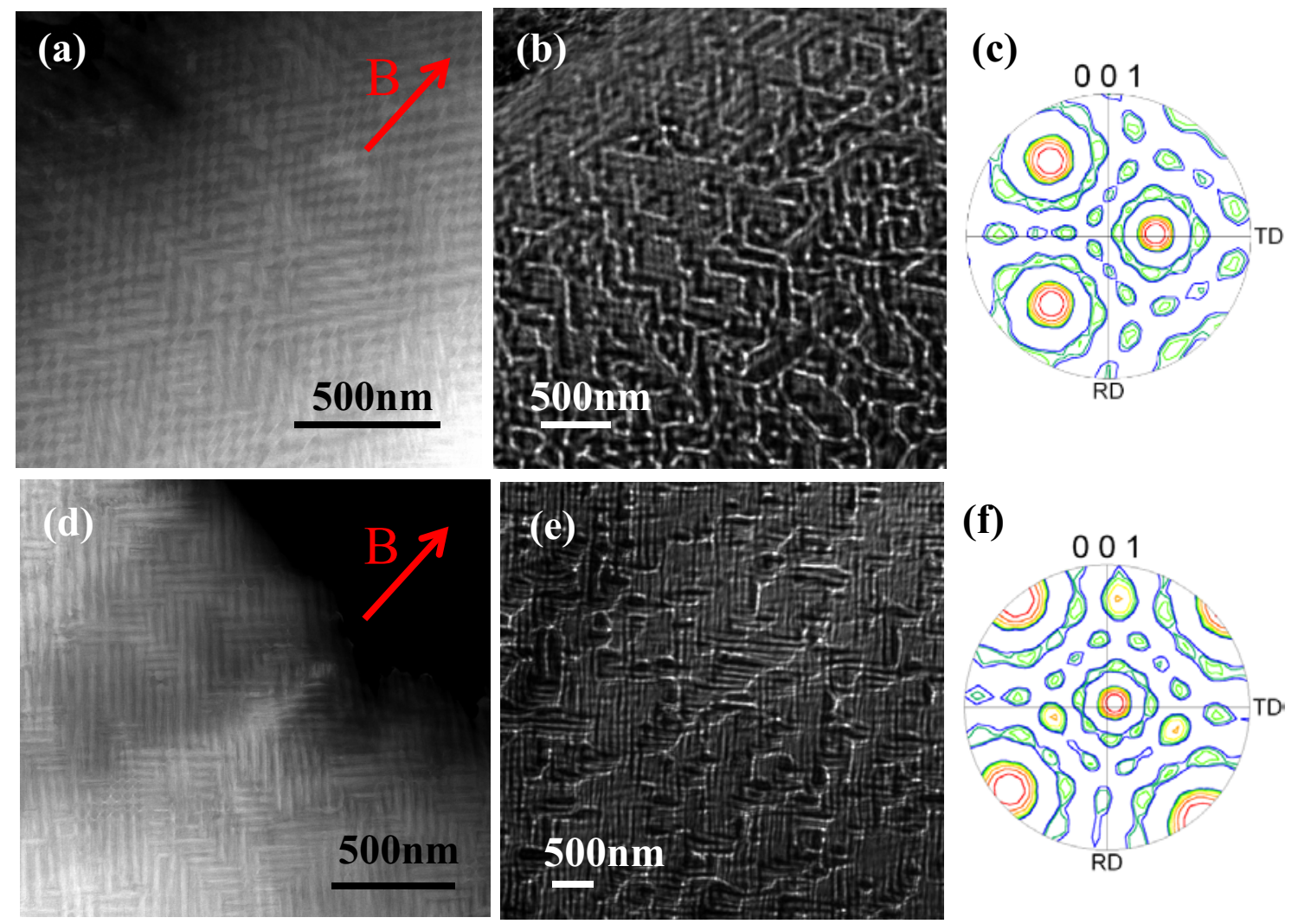

Figure 2. HAADF STEM images ( $a, d)$, Lorentz images $(b, e)$ and pole figures $(c, f)$ of two alnico 8 grains oriented differently relatively to the applied magnetic field direction showing different SD and micro-magnetic domain morphology. 